\title{
Preoperative computed tomography-guided microcoil localization of small peripheral pulmonary nodules: A prospective randomized controlled trial
}

\author{
Richard J. Finley, MD, ${ }^{\mathrm{a}}$ John R. Mayo, MD, ${ }^{\mathrm{b}}$ Kyle Grant, MD, ${ }^{\mathrm{a}}$ Joanne C. Clifton, MSc, ${ }^{\mathrm{a}}$ \\ John English, MD, ${ }^{\mathrm{c}}$ Joyce Leo, MD, ${ }^{\mathrm{c}}$ and Stephen Lam, $\mathrm{MD}^{\mathrm{d}}$
}

\begin{abstract}
Objectives: Growing, small, peripheral, pulmonary nodules in patients at high risk for lung cancer lead to requests for video-assisted thoracoscopic (VATS) resection for pathologic diagnosis. The purpose of this randomized controlled trial was to determine if preoperative localization using percutaneously placed computed tomography (CT)-guided platinum microcoils decreases the need for thoracotomy or VATS anatomic resection (segmentectomy/lobectomy) for diagnosis.
\end{abstract}

\begin{abstract}
Methods: Patients with undiagnosed nodules of $15 \mathrm{~mm}$ or less were randomized to either no localization or preoperative microcoil localization. Coils were placed with the distal end deep to the nodule and the superficial end coiled on the visceral pleural surface with subsequent visualization by intraoperative fluoroscopy and VATS. Nodules were removed by VATS wedge excision using endostaplers. The primary outcome was a VATS wedge excision for pathologic diagnosis of the nodule without the need for either thoracotomy or VATS anatomic resection.
\end{abstract}

Results: Sixty patients were randomized and 56 underwent surgery between March 2010 and June 2012. Twenty-nine underwent microcoil localization and $27 \mathrm{did}$ not. The baseline characteristics (age, sex, forced expiratory volume in the first second of expiration, nodule size/depth) were similar. The coil group had a higher rate of successful diagnosis with VATS wedge resection alone $(27 / 29$ vs $13 / 27 ; P<.001)$, decreased operative time to nodule excision ( $37 \pm 39$ vs $100 \pm 67$ minutes; $P<.001$ ), and reduced stapler firings ( $3.7 \pm 2.0$ vs $5.9 \pm$ $31 ; P=.003)$ with no difference in total costs. Pathologic diagnoses included 14 benign nodules, 32 primary lung malignancies, and 10 metastases. There were no clinically significant complications related to the coil placement or wedge resection.

Conclusions: Preoperative CT-guided microcoil localization decreases the need for thoracotomy or VATS anatomic resection for the diagnosis of small peripheral pulmonary nodules. (J Thorac Cardiovasc Surg 2015;149:26-32)

See related commentry on pages 33-4.

From the Division of Thoracic Surgery, ${ }^{\mathrm{a}}$ Department of Surgery, Department of Radiology, ${ }^{\mathrm{b}}$ Department of Anatomical Pathology, ${ }^{\mathrm{c}}$ and Department of Respiratory Medicine, ${ }^{\mathrm{d}}$ University of British Columbia, Vancouver, British Columbia, Canada.

The study was funded by the British Columbia Lung Association and the VGH/UBC Foundation.

Disclosures: Richard J. Finley: Consultant, Siemens (Advisory Council on Lung Cancer); no salary, royalty, intellectual property rights, consulting fee, honoraria, ownership interest. John R. Mayo: Consultant, Siemens (Advisory Council on Lung Cancer) (lecture fee), and Toshiba Healthcare Canada (lecture fee). Stephen Lam: Consultant (Genome Biomed consultant fees; CSA Medical consultant fees). All other authors have nothing to disclose with regard to commercial support.

Read at the 94th Annual Meeting of The American Association for Thoracic Surgery, Toronto, Ontario, Canada, April 26-30, 2014.

Received for publication April 8, 2014; revisions received Aug 3, 2014; accepted for publication Aug 19, 2014; available ahead of print Oct 5, 2014.

Address for reprints: Richard J. Finley, MD, Division of Thoracic Surgery, Department of Surgery, Vancouver Acute, Vancouver General Hospital Site and the University of British Columbia, Room 7105-2775 Laurel St, Vancouver, BC, Canada V5Z1M9 (E-mail: richard.finley@vch.ca).

$0022-5223 / \$ 36.00$

Crown Copyright $\odot 2015$ Published by Elsevier Inc. on behalf of The American Association for Thoracic Surgery

http://dx.doi.org/10.1016/j.jtcvs.2014.08.055
Detection and safe surgical resection of early lung cancer is the most promising strategy to improve lung cancer survival. The US National Lung Screening Trial showed that screening of patients at high risk for lung cancer with the use of low-dose thoracic computed tomography (CT) reduces mortality from lung cancer by $20 \% .{ }^{1}$ CT detection of nodule growth or an increase in the solid component of semisolid lung nodules in patients at risk for primary or secondary lung cancer raises the possibility of early lung cancer, causing anxiety for both patients and referring clinicians. Unfortunately, confident separation of benign from malignant small lung nodules cannot be reliably achieved using CT criteria $^{2}$ or positron emission tomography (PET)/CT scan analysis. ${ }^{3}$ Pathologic diagnosis using needle or excision biopsy is usually required. Excision biopsy removes the entire nodule at 1 setting and eliminates the sampling error associated with needle biopsy, making it appealing to physicians and patients.

To reduce postoperative morbidity, excision biopsy is often performed using video-assisted thoracoscopic surgery (VATS) techniques. Nodules may be safely resected via minimally invasive techniques without the need for 


\section{Abbreviations and Acronyms \\ $\mathrm{CT}=$ computed tomography \\ PET = positron emission tomography \\ $\mathrm{RCT}=$ randomized controlled trial \\ VATS $=$ video-assisted thoracoscopy}

thoracotomy. Thoracoscopy may facilitate the diagnostic evaluation of potentially malignant lesions by allowing earlier excision biopsy. Patients with benign lesions benefit similarly by being spared an open thoracotomy. However, Suzuki and colleagues ${ }^{4}$ reported that failure to visualize or palpate the nodule during unguided VATS occurred in $54 \%$ of patients, thus requiring conversion to thoracotomy. The conversion rate to thoracotomy increased to $63 \%$ when the nodule was $10 \mathrm{~mm}$ in diameter or less or greater than $5 \mathrm{~mm}$ from the pleural surface. There is particular difficulty in surgically locating low-density semisolid or ground-glass nodules because they are difficult to palpate, even at thoracotomy. To guide VATS wedge resection of these suspicious nodules, we developed a localizing technique that uses preoperative CT guidance to place commercially available fiber-coated microcoils with 1 end deep to the suspicious nodule and the other end in the pleural space. The safety and efficacy of the process was previously evaluated in a pig $\operatorname{model}^{5}$ and in human feasibility trials, ${ }^{6,7}$ which showed $97 \%$ accuracy and a low incidence of complications such as pneumothorax or hemothorax.

Based on this evidence, we carried out a prospective randomized controlled clinical trial. The primary outcome of the study was to determine the accuracy, safety, and total costs of open thoracotomy or VATS anatomic resection (segmentectomy or lobectomy) with preoperative CT-guided microcoil localization and VATS (experimental group) compared with VATS alone (control group) for pathologic diagnosis of small peripheral lung lesions.

\section{METHODS}

The study was approved by the institutional review boards of the University of British Columbia (CREB H09-02265) and Vancouver General Hospital (V10-0028). The randomized controlled trial (RCT) was registered with clinicaltrials.gov (NCT01028417). The CONSORT 2010 checklist of information was used to describe the design, methods, results, and conclusions of this randomized trial. A complete CONSORT diagram (Figure 1) is included. No industry funding was solicited or received for any equipment used in this study.

\section{Sample Size Calculation, Study Duration, and Statistical Analysis}

In our previous phase $1 \mathrm{study}^{7}$ microcoil localization of small peripheral lung nodules enabled fluoroscopy-guided VATS resection of $97 \%$ of the nodules compared with a rate of less than $40 \%$ in historical controls. ${ }^{4}$ The current study was powered to detect an absolute difference of $40 \%$ between the groups in reducing the rate of conversion to open thoracotomy or VATS segmentectomy or lobectomy from $50 \%$ to $10 \%$ for pathologic diagnosis of the target nodule. Twenty-eight patients were required in each group (power $90 \%$; alpha, 0.05 ; beta, 0.10 ). Demographic, preoperative, perioperative, postoperative, pathologic, and cost data were collected. Means and standard deviations were reported for postoperative continuous data and proportions for categorical data. Independent samples $t$ tests were performed on continuous data and $\chi^{2}$ values were calculated for categorical data to test for any significant difference between the experimental and the control groups, including costs.

\section{Randomization}

Computer-generated block randomization was initiated by a data manager in the respiratory research group and placed in individual sealed envelopes, ensuring that both the surgeon and the thoracic research assistant interviewing potential candidates for the study were blind to the randomization code. Each envelope was opened in front of the patient on entry into the study after written informed consent was received.

\section{Study Entry and Exclusion Criteria}

Patients with known pulmonary nodules of $15 \mathrm{~mm}$ or less that were suspicious for cancer, who were referred by family physicians, respiratory physicians, oncologists, and radiologists after July 1, 2009, for possible VATS diagnostic wedge resection were assessed for eligibility. Patients eligible to enter the study had either a pulmonary nodule of 10 to 15 $\mathrm{mm}$, or had a nodule less than $10 \mathrm{~mm}$ that was growing or increasing in density on serial CT. The patient had a contrast-enhanced thin-section CT scan of the chest and upper abdomen, which was assessed by an interventional chest radiologist (J.M.) and a thoracic surgeon (R.F.) with expertise in VATS and open lung resections to determine if the nodules were located in parts of the lung that were amenable to VATS wedge excision. The deepest part of the lesion had to be at least $2 \mathrm{~cm}$ away from major pulmonary arteries and veins to allow safe and adequate VATS excision. All patients were more than 18 years of age and mentally competent to give written informed consent.

Patients were excluded from the trial if they did not consent to participate in the study, if the radiologist and surgeon determined that the nodule was located too centrally to be safely excised using VATS wedge techniques, or if there was a history of previous ipsilateral thoracotomy. Patients with more than 3 nodules and patients with pulmonary hypertension were excluded from the study. If the patient was excluded, they received the current standard treatment, that is, needle biopsy, continued observation of the nodule at 3 to 6 monthly intervals, or excisional surgery (VATS or open thoracotomy).

If the eligibility criteria were met, patients were approached by a trained research assistant in a separate office from the thoracic clinic about study entry. They were informed of the risks and benefits of the procedure and all the alternatives for treatment. Written informed consent was obtained. Patients were then assigned to either the experimental group or the control group.

\section{Surgical Procedure}

Experimental group. As previously described, ${ }^{7}$ preoperative localization of the lung nodule was carried out in the radiology department on the day of surgery using local anesthesia. CT-guided platinum microcoils (VortX-18; Diamond Shape; Boston Scientific, Cork, Ireland) were placed percutaneously through a 22-gauge needle with the distal end deep to the nodule and the superficial end coiled on the visceral pleural surface. The patient was then taken to the operating room, where under general anesthesia with lung isolation, the nodule and coil were removed by wedge excision with endostaplers (Endo GIA II, United States Surgical, Norwalk, Conn; Echelon Endostapler, Ethicon Endo-Surgery, Cincinnati, Ohio) using fluoroscopy and VATS visualization. If the lesion could not be excised using the VATS technique, the patient underwent an open 


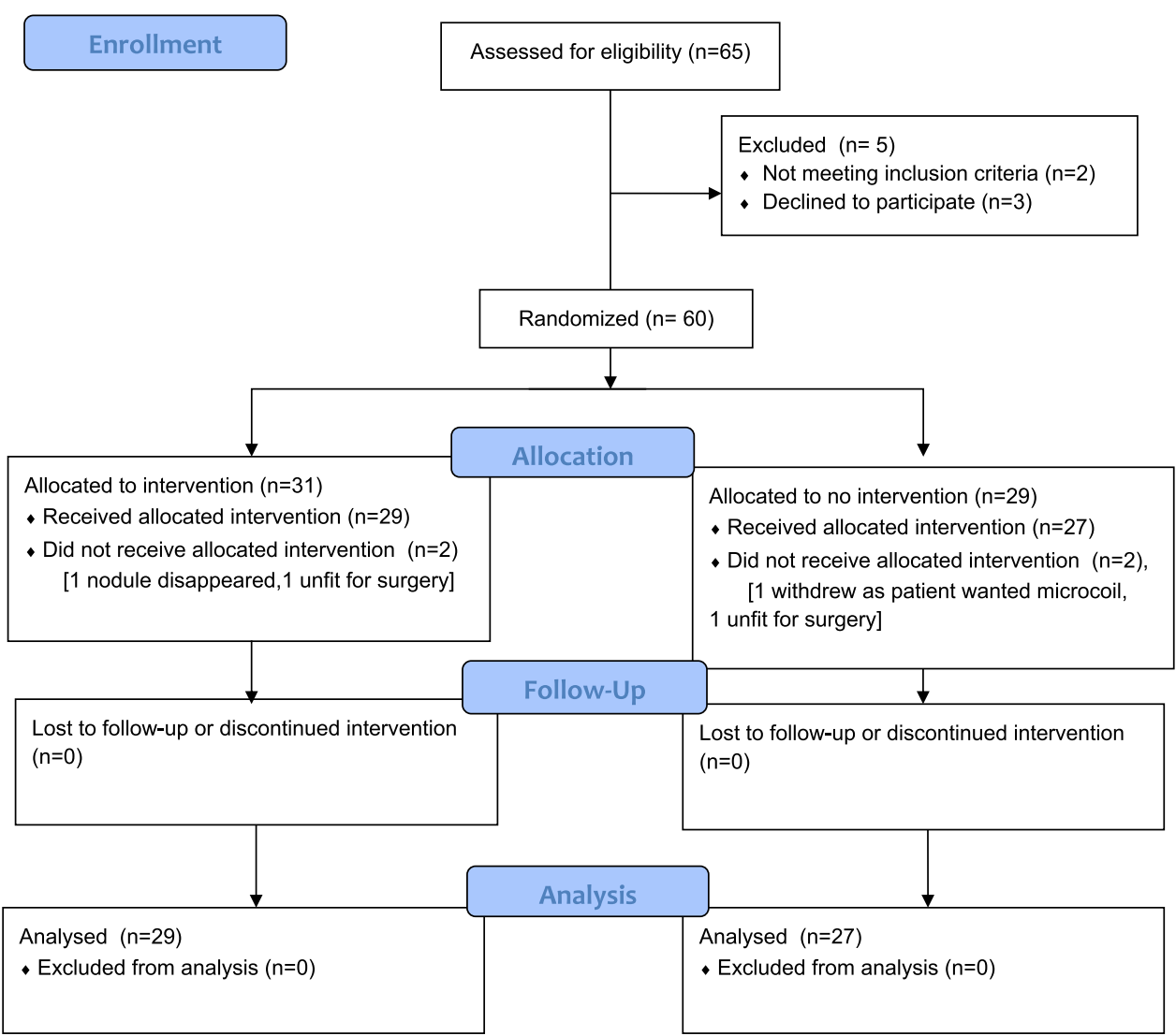

FIGURE 1. CONSORT flow diagram.

rib-spreading thoracotomy for excision of the marked nodule or a VATS anatomic resection (segmentectomy or lobectomy). The indications for these procedures were poor visualization of the lung, diffuse pleural adhesions, uncontrollable bleeding, or inability to completely resect the lesion with the thoracoscopic endostapler.

Control group. Patients in the control group underwent excision of the target lesion using an endoscopic stapling device as directed by a video thoracoscope following the same surgical procedure as above, but without preoperative insertion of the microcoil marker for localization or the use of intraoperative fluoroscopy. The control group received the current standard of care for VATS excision of lung nodules. Standard intraoperative techniques for nodule localization were used. These included visual cues from thoracoscopy (ie, pleural surface deformation) and spatial cues from tactile sensation (finger palpation of deeper nodules as permitted by 10- to 12-mm thoracoscopy port skin incisions). If the lesion could not be excised by the VATS technique, the patient underwent a thoracotomy for wedge excision or a VATS diagnostic anatomic resection. The indications for these procedures were the same as for the experimental group.

\section{Pathologic Analysis}

The specimen was sent for frozen section pathologic examination. A pulmonary pathologist (JE) with 20 years of experience examined the wedge resection according to accepted clinical practice at our institution. The immediate requirements were the assessment of nodule histology (malignant vs benign), and the adequacy of the resection margins. Intraoperative lymph node staging also occurred. Final histologic features of the lung cancer were coded according to the World Health Organization/International Association for the Study of Lung Cancer histologic classification of non-small cell lung cancer. ${ }^{8,9}$ The primary lung cancers were staged using the American Joint Committee on Cancer Cancer Staging Manual (seventh edition). ${ }^{10}$ If the lesion was benign, a metastasis from an extrathoracic malignancy, or the diagnosis was indeterminate, the operation was terminated. If the lesion was a non-small cell cancer of the lung, the patient underwent sampling of lymph nodes and staging. Completion therapeutic lobectomy was carried out unless the patient had inadequate pulmonary function to tolerate a lobectomy, the pathology showed a noninvasive cancer, or the patient had a previous resection of a primary lung cancer.

\section{Patient Safety}

Microcoil localization of the lung nodule took place in the CT suite at Vancouver General Hospital under local anesthetic. If life-threatening complications developed in the CT suite, the patient could be quickly moved to a fully staffed and available operating room.

\section{Outcomes Assessment}

The primary outcome of the study was to determine the rate of open thoracotomy or VATS anatomic resection (segmentectomy or lobectomy) with preoperative CT-guided microcoil localization and VATS (experimental group) compared with VATS alone (control group) for excision and pathologic diagnosis of the target nodule. The outcome is expressed as the percentage of patients undergoing successful and complete excision of the target nodule using VATS without conversion to either an open thoracotomy or VATS anatomic resection. Pathologic 
confirmation of complete nodule excision with negative surgical margins was the gold standard for determining successful excision. The secondary outcomes were preoperative, intraoperative, and postoperative costs from the institutional perspective, and perioperative morbidity and mortality.

\section{Data Collection}

Baseline data were collected using a standardized data form at the initial preoperative visit. Follow-up data were collected at 1 month and 3 months after surgery. The data were validated by the investigators. Demographic data included age, sex, weight, and height. Preoperative variables included smoking history, forced expiratory volume in the first second of expiration, presence of emphysema on the CT scan, and nodule characteristics (size, shape, location, distance from the pleural surface, distance from hilar vessels, Hounsfield units attenuation). Perioperative variables included length of general anesthesia, surgical operating time, CT time, number and type of operating ports, number and type of stapler cartridges required. Intraoperative complications such as staple line failure, bleeding requiring staple line revision, air leak, and incomplete nodule excision were recorded. Pathologic variables included nodule size, gross morphology, histology, as well as minimum and maximum resection margins (millimeters) from the nodule edge to the closest staple line. Postoperative data included postoperative complications (eg, deep venous thrombosis, pneumonia), and length of hospital stay. Each patient had a low-dose CT scan of the chest at least 90 days after the surgery to rule out early cancer recurrences.

\section{Cost of the Procedures}

All direct and professional charges for in-hospital procedures related to excision of the lung nodule were tabulated and analyzed. ${ }^{11}$ Variables specific to the experimental group were the microcoil procedure (microcoil and other procedure-related consumables, CT scanner time, technician's fees, radiologist's fees) and fluoroscopy (fluoroscopy machine and radiology technician in the operating room for the guidance of the microcoil and nodule excision). Cost variables common to both groups relate to the professional fees (anesthesia, surgery), operating room time, consumables, disposables, and reprocessing of instruments. In the postoperative period, costs related to length of stay were tabulated. The per unit time costing variables factor in average capital costs, overhead costs, and labor costs. In the event of failed nodule excision, all costs related to further operations and return to the operating room for second diagnostic procedures were included.

\section{RESULTS}

Patient accrual began in March 1, 2010, and closed in June 20, 2012. Sixty-five patients were assessed for eligibility. Two patients did not meet the inclusion criteria and 3 patients declined to participate. Sixty patients were randomized and 56 underwent surgery between March 2010 and June 2012. The reasons for ineligibility were medically unfit for surgery ( 1 in each group), nodule decreased in size from time of randomization to the date of surgery ( 1 in coil group), patient wanted the microcoil and withdrew from the study ( 1 in the control group). Twenty-nine patients underwent microcoil localization and $27 \mathrm{did}$ not (Figure 1). There were no significant differences in baseline or nodule characteristics between the groups (Table 1).

In the coil group, all coils were successfully placed deep to the lesion and the superficial end was deployed either on the lung surface $(n=23)$ or in lung parenchyma to bracket
TABLE 1. Characteristics of the patients

\begin{tabular}{lcc}
\hline & $\begin{array}{c}\text { Microcoil } \\
(\mathbf{n}=\mathbf{2 9})\end{array}$ & $\begin{array}{c}\text { No microcoil } \\
(\mathbf{n}=\mathbf{2 7})\end{array}$ \\
\hline Median age (y) & 65 & 68 \\
Gender (\% male) & 31 & 44 \\
Smokers (\%) & & \\
$\quad$ Current & 17 & 18 \\
$\quad$ Quit & 66 & 52 \\
$\quad$ Never smoked & 17 & 30 \\
Emphysema (present on CT scan) & 24 & 27 \\
Previous extrathoracic cancers (\%) & 21 & 30 \\
FEV (liters, median) & 2.45 & 2.28 \\
PET positive (\%) & 50 & 48 \\
Nodule size (mm, mean \pm SD) & $12.7 \pm 4$ & $12.4 \pm 5$ \\
Depth in millimeters from pleural surface to (mean \pm SD) & \\
$\quad$ Superficial nodule margin & $10.8 \pm 9.8$ & $9.7 \pm 10.5$ \\
$\quad$ Deep nodule margin & $22.5 \pm 10.3$ & $18.9 \pm 10.8$ \\
Location & & \\
RUL & 7 & 7 \\
RML & 4 & 0 \\
RLL & 7 & 7 \\
LUL & 3 & 3 \\
LLL & 8 & 10 \\
\hline
\end{tabular}

$C T$, Computed tomography; $F E V_{l}$, forced expiratory volume in the first second of expiration; $P E T$, positron emission tomography; $S D$, standard deviation; $R U L$, right upper lobe; $R M L$, right middle lobe; $L U L$, left upper lobe; $L L L$, left lower lobe; $R L L$, right lower lobe.

the nodule $(\mathrm{n}=6)$. No coils were displaced during the procedure. The duration of the preoperative CT-guided microcoil localization procedure was $29 \pm 9$ minutes and intraoperative fluoroscopy time was $0.7 \pm 0.7$ minutes. After coil insertion, no patients developed a hemothorax and 4 patients had a clinically insignificant pneumothorax seen on CT that did not require a chest tube. Compared with the control group, the coil group had a higher rate of successful pathologic diagnosis with VATS wedge resection alone (27 of 29 vs 13 of $27 ; P<.001$ ), decreased operative time to nodule excision $(37 \pm 39$ vs $100 \pm 67$ minutes; $P<.001)$, and a reduced number of intraoperative stapler firings $(3.7 \pm 2.0$ vs $5.9 \pm 3.1 ; P=.003)$. In the coil group, 2 patients had VATS anatomic resections for diagnosis. The other 27 patients had wedge resection alone (17) or wedge for diagnosis plus VATS completion lobectomy (10). In the control group, 14 patients had nodules that could not be excised by VATS wedge resection alone. To establish the histologic diagnosis of the nodule, 2 patients needed a second VATS wedge at the initial operation, 1 patient needed a thoracotomy and wedge resection, 8 patients needed either a VATS segmentectomy (3) or lobectomy (5), and 1 patient had no nodule in the wedge at final pathology and was too ill to have a second surgery. Two patients required subsequent operation to remove the nodule. In the second surgery, 1 of these patients underwent preoperative CT-guided microcoil localization and wedge resection and the other underwent a VATS lobectomy. 
Pathologic diagnosis included benign (14), metastases (10), and primary lung malignancy (32). The benign lesions consisted of granulomas (6), localized pneumonitis (2), hemangioma (1), aspiration (1), adenomatous hyperplasia (1), intrapulmonary lymph node (1), and fibrosis (1). The metastasis consisted of colon (7), kidney (1), thyroid (1), and cervix (1). Primary adenocarcinomas were observed in 30 patients with the following subclassification: adenocarcinoma in situ (3), minimally invasive carcinoma (8), and invasive adenocarcinoma (19). Two patients had squamous cell cancer. Thirty patients had stage 1 disease and 2 patients had stage 2 disease. The surgeries performed on the 32 patients with primary lung cancer included wedge alone (17), segmentectomy (3), diagnostic wedge with completion therapeutic lobectomy (7), and diagnostic lobectomy (5). There were no deaths or cancer recurrences in the first 90 days after the surgery. There were no clinically relevant wound infections, pneumonia, empyema, or myocardial infarctions in the postoperative period. One patient in each of the groups had a prolonged air leak. The mean hospital length of stay for all procedures in the microcoil group was $4.3 \pm 2.6$ days (median, 3.0 days; range, 2-12 days) versus $5.0 \pm 2.4$ days (median, 5.0 days; range, 2-12 days) in the control group. There was no significant difference between the microcoil and control groups for all costs (in Canadian dollars) required for VATS wedge excision alone $(\$ 4301 \pm \$ 1429$ [median, $\$ 3901$; range, \$2013-\$7641] vs $\$ 4365 \pm \$ 1250$ [median, $\$ 4156$; range, \$1968-\$5840]) and for all procedures for complete nodule excision $(\$ 4903 \pm \$ 2518$ [median, $\$ 3930$; range, \$2366-\$11,952] vs \$5803 \pm \$2615 [median, \$5363; range, \$1975-\$11,282]) (Table 2).

\section{DISCUSSION}

Data from this RCT has confirmed the results of previous

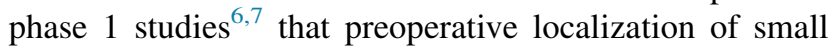
peripheral pulmonary nodules $(\leq 15 \mathrm{~mm})$ using percutaneous CT-guided microcoil localization followed by fluoroscopy-guided VATS resection increases the likelihood of complete excision of the target nodule for

TABLE 2. Cost details (Canadian dollars) for 56 patients for all procedures required for complete nodule excision

\begin{tabular}{lcc}
\hline \multicolumn{1}{c}{ Cost details } & $\begin{array}{c}\text { Microcoil } \\
(\mathbf{n = 2 9 )}\end{array}$ & $\begin{array}{r}\text { No microcoil } \\
(\mathbf{n = 2 7 )}\end{array}$ \\
\hline Ward & 2155 & 2500 \\
Cost of microcoil procedure* & 567 & 26 \\
Anesthesia* & 164 & 447 \\
Fluoroscopy time* & 122 & 5 \\
Operating time* & 121 & 331 \\
Handles and cartridges* & 1137 & 1626 \\
Surgeon* & 634 & 866 \\
Total cost (nonsignificant) & $4903 \pm 2518$ & $5803 \pm 2615$ \\
\hline$P<.05$. & &
\end{tabular}

pathologic diagnosis using VATS without conversion to an open thoracotomy or VATS anatomic resection (segmentectomy or lobectomy). In the coil group, 27 of $29(93 \%)$ patients had a complete resection of the nodule for diagnosis with VATS wedge resection alone, whereas only 13 of $27(48 \%)$ patients without image guidance had a successful diagnostic VATS wedge resection alone. In the control group, one half of the patients had to undergo more invasive or additional procedures in order to establish the diagnosis, thus adding to the morbidity and cost of the procedure. There was no significant difference between the 2 groups for total costs. The expense of the insertion and intraoperative localization of the microcoil was offset by the price of increased operating time and disposable costs and additional procedures used to establish the pathologic diagnosis of the nodule in the patients with no localization procedure.

These resection rates without localization techniques are similar to those of Suzuki and colleagues ${ }^{4}$ and can be explained by the difficulty in visualizing or palpating semisolid or ground-glass opacity nodules at the time of VATS. These nonsolid nodules, especially those of the semisolid variety, have a higher probability of malignancy compared with solid nodules and are more commonly falsely negative on PET. ${ }^{3}$ The localization technique used in this study is particularly beneficial in localizing this type of subpleural nodule. Several small nodule localization techniques have been developed in an attempt to guide VATS resection. Finger palpation of the nodule has a localization percentage less than $40 \%,{ }^{12}$ Intraoperative ultrasonography ${ }^{12}$ improves this localization rate but is less reliable in patients with emphysema. Hook wire localization $^{13}$ is associated with a high frequency of pneumothorax. Injection of methylene blue, ${ }^{14}$ lipiodol, ${ }^{15}$ or radioisotope ${ }^{16}$ improves VATS wedge resection rates but can be associated with venous embolization or diffusion of the injected substance preventing accurate guidance. The low rates of procedural complications observed using percutaneous CT-guided microcoil localization, including minor pneumothorax requiring no chest tube $(13 \%)$, hemothorax $(0 \%)$, coil displacement $(0 \%)$, and unsuccessful VATS resection $(7 \%)$, suggest a favorable safety and success profile compared with the other techniques.

The preoperative CT-guided placement of the coil deep to the lesion resulted in clear pathologic margins and no early local recurrences with resection of the coil and the malignant nodule. Although the lesion was directly punctured using the localization technique, this did not impede histopathologic processing or interpretation of the specimens. The overall rate of $25 \%$ benign lesions in this RCT is similar to other studies documenting the excision of small nodules. ${ }^{12,16}$ Ninety-four percent of patients with primary lung cancer had stage 1 disease and only $63 \%$ of the adenocarcinomas were invasive. The surgeries 
performed on the 32 patients with primary lung cancer included wedge alone (17), segmentectomy (3), diagnostic wedge with completion therapeutic lobectomy (7), and diagnostic lobectomy (5). Long-term follow-up will be needed to determine the efficacy of these procedures in curing the cancers.

In our previous reports, the end point used was failure to visualize or palpate the nodule at VATS, requiring a treatment change to thoracotomy. With the evolution of anatomic lung resection from open thoracotomy to VATS in our center, we modified the primary end point in this RCT to the percentage of patients undergoing complete excision of the target nodule using VATS without conversion to an open thoracotomy or VATS segmentectomy or lobectomy. This modification was submitted to the Ethics Review Board and approved.

This study was limited in that all nodule resections were performed at 1 academic teaching center. One interventional chest radiologist with 25 years of experience performed all CT localizations. Some peripheral nodules included in this study might have been successfully resected without any localization techniques by using a larger access incision to improve tactile localization of the nodule. Furthermore, the nodules could be excised using VATS segmentectomy or lobectomy, sparing the need for localization. However, 1 of the patients in the control group underwent a formal VATS segmentectomy for a semisolid lesion and had cancer at the resection margin on final pathology requiring a second operation and completion lobectomy. A high degree of cooperation between the radiology and surgery departments allowed coordination of the CT localizations in the morning and the VATS excisions in the afternoon. This technique can safely be applied in hospitals with experienced interventional chest radiologists, pulmonary pathologists, and thoracic surgeons with experience in intraoperative fluoroscopy and VATS pulmonary resections.

In summary, this study found that preoperative CT-guided microcoil localization and fluoroscopy-guided VATS excision increased the success rate of VATS excision of small lung nodules for pathologic diagnosis to $93 \%$ compared with $48 \%$ without guidance. The use of preoperative CT-guided microcoil localization decreased the number and extent of procedures, but did not increase patient risk or hospital costs for histologic diagnosis of the target lesion. The use of this technique may help to address the diagnostic dilemma of increasing numbers of small peripheral lung nodules that are detected incidentally or found at serial chest $\mathrm{CT}$ to be growing or increasing in density.

R.J. Finley acknowledges the support of his colleagues, Dr Ken Evans and Dr John Yee, who provided clinical coverage while he conducted this work. We thank Dr Annette McWilliams for her referral and follow-up of patients.

\section{References}

1. Aberle DR, Adams AM, Berg CD, Black WC, Clapp JD, Fagerstrom RM, et al; National Lung Screening Trial Research Team. Reduced lung cancer mortality with low-dose computed tomographic screening. N Engl J Med. 2011;365: 395-409.

2. Libby DM, Smith JP, Altorki NK, Pasmantier MW, Yankelevitz D, Henschke CI Managing the small pulmonary nodule discovered by CT. Chest. 2004;125: 1522-9.

3. Khalaf M, Abdel-Nabi H, Baker J, Shao Y, Lamonica D, Gona J. Relation between nodule size and 18FDG-PET SUV for malignant and benign pulmonary nodules. J Hematol Oncol. 2008;1:13.

4. Suzuki K, Nagai K, Yoshida J, Ohmatsu H, Takahashi K, Nishimura M, et al. Video-assisted thoracoscopic surgery for small indeterminate pulmonary nodules: indications for preoperative marking. Chest. 1999;115:563-8.

5. Jangra D, Powell T, Guerra L, Guerra HL, Clifton J, Coxson HO, et al. CT-directed microcoil localization of small peripheral lung nodules: a feasibility study in pigs. J Invest Surg. 2005;18:265-72.

6. Powell T, Jangara D, Clifton J, Lara-Guerra H, Church N, English J, et al Peripheral lung nodules: fluoroscopically guided video-assisted thoracoscopic resection after computed tomography-guided localization using platinum microcoils. Ann Surg. 2004;240:481-8.

7. Mayo J, Clifton J, Powell J, English J, Evans KG, Yee J, et al. Lung nodules: CT-guided placement of microcoils to direct video-assisted thoracoscopic surgical resection. Radiology. 2009;250:576-85.

8. Travis WD, Brambilla E, Müller-Hermelink HK, Harris CC, eds. World Health Organization Classification of Tumours. Pathology and Genetics of Tumors of the Lung, Pleura, Thymus and Heart. Lyon, France: IARC; 2004.

9. Travis WD, Brambilla E, Noguchi M, Nicholson AG, Geisinger KR, Yatabe Y, et al. International Association for the Study of Lung Cancer/American Thoracic Society/European Respiratory Society international multidisciplinary classification of lung adenocarcinoma. J Thorac Oncol. 2011;6:244-85.

10. Edge SB, Byrd DR, Compton CC, Fritz AG, Greene FL, Trotti A, eds. AJCC Cancer Staging Manual. 7th ed. New York: Springer; 2010:253-70.

11. Ramsey SD, McIntosh M, Sullivan SD. Design issues for conducting cost effectiveness analyses alongside clinical trials. Annu Rev Public Health. 2001; 22:129-41.

12. Khereba M, Ferraro P, Duranceau A, Martin J, Goudie E, Thiffault V, et al Thoracoscopic localization of intraparenchymal pulmonary nodules using direct intracavitary thoracoscopic ultrasonography prevents conversion of VATS procedures to thoracotomy in selected patients. J Thorac Cardiovasc Surg. 2012;144:1160-5.

13. Miyoshi K, Toyooka S, Gobara H, Oto T, Mimura H, Sano Y, et al. Clinical outcomes of short hook wire and suture marking system in thoracoscopic resection for pulmonary nodules. Eur J Cardiothorac Surg. 2009;36:378-82.

14. McConnell PI, Feola GP, Meyers RL. Methylene blue-stained autologous blood for needle localization and thoracoscopic resection of deep pulmonary nodules. J Pediatr Surg. 2002;37:1729-31.

15. Watanabe K, Nomori H, Ohtsuka T, Kaji M, Naruke T, Suemasu K. Usefulness and complications of computed tomography-guided lipiodol marking for fluoroscopy-assisted thoracoscopic resection of small pulmonary nodules: experience with 174 nodules. J Thorac Cardiovasc Surg. 2006;132:320-4.

16. Gonfiotti A, Davini F, Vaggelli L, De Francisci A, Caldarella A, Gigli PM, et al Thoracoscopic localization techniques for patients with solitary pulmonary nodule: hookwire versus radio-guided surgery. Eur J Cardiothorac Surg. 2007 32:843-7.

\section{Discussion}

Dr Michael J. Liptay (Chicago, Ill). Thank you, Dr Finley, for that elegant presentation of a very innovative technique to assist VATS surgeons when pressed into service for these often very difficult to find small and semisolid lesions.

I have a few questions. First, I had the privilege of reading the manuscript, but you did not mention it much here. What do you think explains the less than $50 \%$ ability for a VATS wedge in the control group? I recognize that these lesions are hard to find, but is there a technique that you are limiting it? Are you using a larger 
access incision? I read somewhere in the manuscript that the ports were $10 \mathrm{~mm}$

Dr Finley. Before the operation I reviewed the location and pulmonary landmarks of the lesion with the chest radiologist. In the control group, I was able to excise $47 \%$ of these lesions compared with $93 \%$ in the coil group by palpation with my finger through 1 of the $10-\mathrm{mm}$ ports. Difficulty in feeling and excising these small subpleural semisolid and ground-glass opacity lesions by the VATS technique has also been described by other surgeons.

Dr Liptay. Correct. I mention that only because if you are limiting all the ports to $10 \mathrm{~mm}$, if you are planning on a completion lobectomy, you are going to extend 1 of those ports. The principles of VATS surgery are primarily non-rib spreading. So if you are using a little bit bigger port, sometimes you might have a little better chance to access those nodules.

You also mentioned that there is no added cost to the procedure and basically the cost of the microcoiling was offset by using less staple loads and shorter cases, but we also noted that there were more wedge resections in the coil group, even for cancer. Seventeen of the 29 patients had wedge only for cancer, and they seemed on your demographics to be healthy patients, 65 years old, good $\mathrm{FEV}_{1}$. Can you explain that?

Dr Finley. In the coil group there were 16 patients with primary lung cancer, 8 of whom underwent lobectomy. The other patients had noninvasive cancer or poor pulmonary function or previous pulmonary resections or were of Asian descent and prone to develop second primaries. The control group needed more lobectomies and segmentectomies in order to make the diagnosis because of poor localization, thus increasing the cost.

Dr Liptay. My last question is a practical one. Is it feasible at the time of the coil placement to perform fine-needle aspiration?

Dr Finley. Yes, it is feasible to do fine-needle aspiration at the time of the coil placement. However, we have found a high false-negative rate and waiting for the cytology results is costly.

Dr Liptay. Thanks for the privilege. I appreciate it.

Dr Finley. Thank you.

Dr Thomas A. D'Amico (Durham, NC). Rich, I really applaud your group completing this prospective randomized study.

I just have a few quick questions. Did getting a biopsy preclude being on the study? So if you were there in a procedure, and you knew the needle was in the nodule, were you not allowed to get a biopsy to be on the study?

Dr Finley. Many of these patients had previous CT-guided needle biopsy attempts that were nondiagnostic.

Dr D'Amico. Attempts. That's fine.
Was frozen section ever wrong in the operating room?

Dr Finley. Frozen sections histopathology to diagnose invasive cancer in the ground-glass opacities or the semisolid nodules were difficult. Preoperative coil insertion facilitated the localization and accurate diagnosis of the frozen section of the lesion by the pathologist. In the control group, 2 patients required further surgery as a result of an inaccurate frozen section diagnosis.

Dr D'Amico. So that would allow you to then have a different strategy. If you are not going to trust the frozen section anyway, why don't you just go right to segmentectomy on all of these, and if you had done that, what would the results have been in the control group? I'm sure it is in the manuscript, but how many in the control group were not cancer, and so if you had gone right to a segmentectomy, that would have been the wrong thing for that patient?

Dr Finley. If we had gone right to segmentectomy in the control group, about $20 \%$ were benign. In the control group, 1 patient had a primary segmentectomy for diagnosis of a ground-glass opacity. The final pathology showed that we missed part of the tumor because we did not mark the deep edge of the tumor with a microcoil. The patient required a completion lobectomy at a second operation. The 1 advantage of the microcoil localization technique is that, if we have removed the coil, we have a clear deep margin of the lesion.

Dr D'Amico. And if you are doing this, you find it with the coil, you are going to do a wedge and you realize that the wedge lines would cross segment lines, do you just go right to a segmentectomy? Do you do the wedge and deal with it later and do a lobe? How do you deal with that?

Dr Finley. Usually we do a wedge resection followed by a completion therapeutic lobectomy if indicated.

Dr David P. Mason (Cleveland, Ohio). Fluoroscopy, it is in full lateral; is that right?

Dr Finley. We use three-dimensional full range fluoroscopy.

Dr Mason. The patient is in full lateral?

Dr Finley. Yes, a full lateral position, set up for a VATS resection.

Dr Mason. I am not that good on fluoro. It just seems like fluoroscopy of a three-dimensional structure to find something like that could be challenging, but you did it quickly. I do not know if there are any tips that you might be able to give.

Dr Finley. The important steps for an accurate and quick location of the nodule are preoperative planning with the radiologist and training of the surgeon in safe and effective intraoperative fluoroscopy. 